Article

\title{
The Correlates of Post-Traumatic Stress Disorder in Ambulance Personnel and Barriers Faced in Accessing Care for Work-Related Stress
}

\author{
Itumeleng Ntatamala *(D) and Shahieda Adams (D)
}

check for updates

Citation: Ntatamala, I.; Adams, S The Correlates of Post-Traumatic Stress Disorder in Ambulance Personnel and Barriers Faced in Accessing Care for Work-Related Stress. Int. J. Environ. Res. Public Health 2022, 19, 2046. https:// doi.org/10.3390/ijerph19042046 Academic Editors: Saloshni Naidoo and Rajen Naidoo

Received: 5 January 2022

Accepted: 7 February 2022

Published: 11 February 2022

Publisher's Note: MDPI stays neutral with regard to jurisdictional claims in published maps and institutional affiliations.

Copyright: (c) 2022 by the authors. Licensee MDPI, Basel, Switzerland. This article is an open access article distributed under the terms and conditions of the Creative Commons Attribution (CC BY) license (https:// creativecommons.org/licenses/by/ $4.0 /)$.
Division of Occupational Medicine and Centre for Environmental and Occupational Health Research, School of Public Health and Family Medicine, University of Cape Town, Cape Town 7925, South Africa; shahieda.adams@uct.ac.za

* Correspondence: itumeleng.ntatamala@uct.ac.za; Tel.: +27-21-40666650

\begin{abstract}
We investigated factors associated with increased risk for post-traumatic stress disorder (PTSD) in ambulance personnel and the barriers faced in accessing support for work-related stress (WRS). A cross-sectional study of 388 ambulance personnel used self-administered questionnaires to assess for PTSD and level of occupational stressors: Impact of Event Scale-Revised, Emergency Medical Services (EMS) Critical Incident Inventory, EMS Chronic Stress Questionnaire, SF-36 Quality of Life and the Connor-Davidson Resilience Scale. The prevalence of PTSD in the study population was $30 \%$. The participants were predominantly female $(55 \%)$, with a median age of 38 (IQR; $31-44$ ) years. PTSD was associated with smoking (OR $=1.76,95 \% \mathrm{CI}$ : 1.05-2.95), illicit drug use (OR $=16.4,95 \% \mathrm{CI}$ : 1.87-143.86) and problem drinking ( $\mathrm{OR}=3.86,95 \% \mathrm{CI}$ : 1.80-8.23). A self-reported mental health condition $(\mathrm{OR}=3.76,95 \% \mathrm{CI}: 1.96-7.21)$, being treated for a medical condition (OR $=1.95,95 \% \mathrm{CI}: 1.22-3.11)$, exposure to critical incident stress (OR $=4.27,95 \%$ CI: $2.24-8.15)$ and chronic WRS (OR $=4.46,95 \%$ CI: 1.93-10.31) were associated with PTSD risk. Barriers to seeking help included concerns that services were not confidential and the negative impact on the participant's career. The increased levels of WRS, strong associations with substance use and barriers to accessing care offer starting points for workplace interventions to reduce the impact of PTSD in ambulance personnel.
\end{abstract}

Keywords: paramedic; ambulance personnel; occupational; work-related stress; PTSD; post-traumatic stress; barriers

\section{Introduction}

Exposure to occupational stressors, such as verbal and physical assault and responding to traumatic scenes including motor vehicle accidents or critically ill patients, has been linked to the development of stress reactions such as post-traumatic stress disorder (PTSD) and other trauma-related conditions in frontline emergency workers [1-7].

Studies have found an increased PTSD prevalence in ambulance personnel compared with the general population and other emergency workers. A pooled PTSD prevalence of $10 \%$ was found in emergency workers compared to $1.3-3.5 \%$ in the general population [8]. PTSD prevalence was highest among ambulance personnel (14.6\%, 95\% CI: 8.80-20.30) compared to fire fighters $(7.30 \%, 95 \%$ CI: $3.60-11.00)$ and police officers $(4.70 \%, 95 \%$ CI: $1.20-8.30$ ). Others have reported an estimated PTSD prevalence of $11 \%$ among ambulance personnel worldwide, which was higher than that of the general population [9]. Possible explanations for increased PTSD prevalence include the fact that ambulance personnel respond to a greater number of calls than other emergency workers, have greater personal contact with patients and their families, have more sustained work pressure when on duty and often work with limited resources in strained health systems [8-12]. Crosssectional studies have identified demographic factors such as younger age, female gender, educational level, marital status and living alone as correlates for PTSD in ambulance 
personnel [13-16]. Other significant correlates include the frequency and intensity of exposure to acute and chronic stress [17-19], lack of social support from colleagues and management $[12,20]$, pre-existing psychiatric illness and poor coping style $[5,16]$.

The prevalence of PTSD in South African ambulance personnel was reported as $6.67 \%$ (in 2005) and 16\% (in 2014) [11,12]. Ward et al. [6] found that South African ambulance, fire and sea rescue service workers experienced more exposure to critical events and suffered higher rates of general psychopathology compared to workers in high-income countries.

Despite evidence of increasing exposure to critical events, there is a paucity of research in South Africa documenting trends in the prevalence of PTSD and factors associated with its development in ambulance personnel. Little is also known about what barriers they face in accessing support for work-related stress. This study aimed to determine factors associated with an increased risk for PTSD and the preferred supportive measures for workrelated stress (WRS) in ambulance personnel in the Western Cape Province, South Africa.

\section{Materials and Methods}

\subsection{Study Design, Population and Sampling}

A cross-sectional study of ambulance personnel employed by the Western Cape Department of Health situated at each of 50 ambulance bases was conducted between 15 November 2019 and 17 January 2020. An electronic questionnaire was distributed to all of the ambulance personnel in operational (providing direct clinical care) and support service roles. Both electronic and hard copy versions of the questionnaires were provided based on the participant's choice. No exclusion criteria were applied in this study. All of the participants were required to provide informed consent prior to participating in the study. The study was approved by the University of Cape Town's Human Research Ethics Committee (HREC 517/2019).

\subsection{Measurements}

\subsubsection{Author Questionnaire}

The author questionnaire gathered self-reported sociodemographic data including age, gender, language, level of education, marital status, living status and occupational data such as 'job category, length of service, location of work'. Mental health and medical history data were obtained, including 'own/family history of mental health diagnosis, treatment for medical or mental health condition'. Smoking, alcohol, illicit and prescription drug use history was also solicited. The CAGE questionnaire was used to assess problem drinking (score of $\geq 2 / 4$ indicative of problem drinking). Questions on barriers faced in seeking help for work-related stress and preferred sources of support were included.

\subsubsection{Impact of Event Scale-Revised (IES-R)}

The Impact of Event Scale-Revised (IES-R) was used to assess post-traumatic stress symptoms experienced (during the past seven days) in relation to the most troubling critical incident encountered in the line of duty in the past 6 months. This validated and widely used 22-item scale contains questions that cover three PTSD symptom clusters [21,22]. Symptoms are scored on a five-point Likert scale (0-4) in response to the most troubling critical or traumatic event/s experienced in the workplace. The higher scores obtained indicated greater symptomatic stress, and a cut-off score of above 35 was used as indicative of a PTSD 'case' [22].

\subsubsection{Connor-Davidson Resilience Scale (CD-RISC)}

The validated 10-item Connor-Davidson Resilience Scale (CD-RISC) was used to measure resilience in the ambulance personnel. The participant's adaptive behaviours in stressful situations were used to identify resilient characteristics and these were scored on a 5-point Likert scale. Higher scores indicate greater resilience. The CD-RISC scale has been noted as one of the most reliable and efficient measures of resilience with an internal 
consistency of between 0.7 from 0.9 [23]. Written permission to use the scale was obtained from the authors.

\subsubsection{EMS Critical Incident Inventory (CII)}

The Emergency Medical Services (EMS) Critical Incident Inventory (CII) developed by Ward et al. [6] and Donnelly and Bennett [24] was used to assess exposure to critical incidents encountered in the line of duty and to obtain an indication of the frequency of exposure in the past 6 months. The activities encountered during ambulance work are contained in the CII.

\subsubsection{EMS Chronic Stress Questionnaire (EMS-CSQ)}

The EMS Chronic Stress Questionnaire (EMS-CSQ) is a validated tool used to assess the exposure to and perceptions of chronic stress experienced by ambulance personnel $[7,25]$. The tool assesses both organizational and operational types of chronic workplace stress, with each scale consisting of 10 questions. It defines operational stress as that associated with structural elements of working in EMS such as shift work, risk of being injured in the line of duty and fatigue. Organizational stress includes factors associated with organizational culture, such as conflict with supervisors and staff shortages.

\subsubsection{SF-36 Quality of Life Questionnaire (SF-36 QOL)}

Reduced quality of life and interference with occupational functioning due to emotional problems such as feeling depressed, anxious or stressed was measured using three questions from the SF-36 Quality of Life questionnaire (SF-36 QOL). This validated tool is widely used to measure general health status in emergency workers and the general population [26,27].

\subsection{Statistical Analysis}

Stata 14.0 statistical computer software (StataCorp, College Station, Texas, USA) as was used for the analysis of all of the data collected. The main associations of interest were between the explanatory variables (socio-demographic, occupational and environmental risk factors, general and mental health status) and post-traumatic stress disorder as an outcome variable. Bivariate analysis was then undertaken to examine the correlation between post-traumatic stress symptom scores with various workplace and environmental risk factors. These variables were then analyzed using unadjusted logistic regression with a calculation of the odds ratio (OR) followed by multivariate regression analysis, with adjustment for age, gender and educational level. To further explore the relationship between PTSD and continuous variables (resilience, chronic stress and critical incident stress scores), three tertiles of these variables were created (high, moderate and low) while four categories were created for age and SF-36 QOL scores.

\section{Results}

Of the 2000 distributed questionnaires, 478 were returned representing a $24 \%$ response rate. However, two were duplications and 88 were incomplete and could not be used in the analysis. Only 388 entries were therefore analyzed in this study.

\subsection{Demographic and General Health Qualities of Participants}

The demographic characteristics of the study population are outlined in Table 1 . The participants were predominantly female (55\%), with median age 38 (IQR: $31-44$ ) years and most had a professional qualification (83\%). The majority lived with family or friends (79\%). 
Table 1. Demographic and occupational qualities of participants $(n=388)$.

\begin{tabular}{|c|c|c|}
\hline Participant Characteristics & Frequency $(n)$ & Percentage (\%) \\
\hline \multicolumn{3}{|l|}{ Sex } \\
\hline Male & 175 & $45.10 \%$ \\
\hline Female & 213 & $54.90 \%$ \\
\hline \multicolumn{3}{|l|}{ Age } \\
\hline Age, years $(n=339)$ & $38(31-44)$ & \\
\hline \multicolumn{3}{|l|}{ Home language } \\
\hline Afrikaans & 178 & $45.88 \%$ \\
\hline English & 122 & $31.44 \%$ \\
\hline IsiXhosa & 84 & $21.65 \%$ \\
\hline Other & 4 & $1.03 \%$ \\
\hline \multicolumn{3}{|l|}{ Level of education } \\
\hline Basic (Grade 1-12) & 35 & $9.02 \%$ \\
\hline Certificate & 284 & $73.20 \%$ \\
\hline Diploma & 45 & $11.60 \%$ \\
\hline Degree & 24 & $6.19 \%$ \\
\hline \multicolumn{3}{|l|}{ Marital status } \\
\hline Never married & 172 & $44.33 \%$ \\
\hline Married & 174 & $44.85 \%$ \\
\hline Divorced or separated & 36 & $9.28 \%$ \\
\hline Widowed & 6 & $1.55 \%$ \\
\hline \multicolumn{3}{|l|}{ Place of living } \\
\hline Rural & 153 & $39.43 \%$ \\
\hline Urban & 235 & $60.57 \%$ \\
\hline \multicolumn{3}{|l|}{ Current living status } \\
\hline Alone & 81 & $20.88 \%$ \\
\hline Family or friends & 307 & $79.12 \%$ \\
\hline \multicolumn{3}{|l|}{ Occupational status } \\
\hline Professional health qualification & 322 & $82.99 \%$ \\
\hline \multicolumn{3}{|l|}{ Job category } \\
\hline Operational services & 277 & 71.39 \\
\hline Ambulance Services & 233 & $60.05 \%$ \\
\hline HealthNet (Non-Emergency Transport) & 19 & $4.90 \%$ \\
\hline Rescue Services & 25 & $6.44 \%$ \\
\hline Support services & 111 & 28.61 \\
\hline Call Centre & 88 & $22.68 \%$ \\
\hline Managers, Admin, Finance \& Other & 23 & $5.93 \%$ \\
\hline Years employed in current role $(n=383)$ & $7.7(3.4-2.2)$ & \\
\hline Years employed in a health environment $(n=383)$ & $9.9(5.8-15)$ & \\
\hline \multicolumn{3}{|l|}{ Place of work } \\
\hline Urban & 184 & $47.42 \%$ \\
\hline Rural & 204 & $52.58 \%$ \\
\hline Job/role change at work in the past 5 years & 98 & $25.26 \%$ \\
\hline \multicolumn{3}{|l|}{ Average hours worked per week } \\
\hline $30-40 \mathrm{~h}$ & 122 & $31.44 \%$ \\
\hline $41-50 \mathrm{~h}$ & 121 & $31.19 \%$ \\
\hline $51-60 \mathrm{~h}$ & 130 & $33.51 \%$ \\
\hline $61-70 \mathrm{~h}$ & 15 & $3.87 \%$ \\
\hline \multicolumn{3}{|l|}{ Monthly salary (South African Rands) } \\
\hline ZAR 0-10,000 & 41 & $10.57 \%$ \\
\hline ZAR $10,001-20,000$ & 238 & $61.34 \%$ \\
\hline ZAR 20,001 and above & 109 & $28.09 \%$ \\
\hline
\end{tabular}

Data are presented as \% or median (interquartile range) unless otherwise indicated.

A third of participants (30\%) were current smokers while half (52\%) currently used alcohol (Table 2). Of those who currently used alcohol $(n=200), 27 \%$ had problem drinking based on their CAGE score. Males had significantly more problem drinking than females $(32.03 \%$ vs. $18.06 \%$; $p$-value $=0.03)$. Only $3 \%$ of all of the participants indicated current use of illicit/non-prescription drugs. 
Table 2. Frequency and distribution of general health specific variables $(n=388)$.

\begin{tabular}{ccc}
\hline Participant Characteristics & Frequency (n) & Percentage (\%) \\
\hline Smoking history & & \\
Current & 118 & $30.41 \%$ \\
Previous smoker & 33 & $9.02 \%$ \\
Non-smoker & 235 & $60.57 \%$ \\
Alcohol use & & \\
Current & 200 & $51.55 \%$ \\
Previous alcohol use & 78 & $20.10 \%$ \\
No alcohol use & 110 & $28.35 \%$ \\
Alcohol misuse & & \\
CAGE score: 0-1 & 146 & $73.00 \%$ \\
CAGE score: 2-4 & 54 & $27.00 \%$ \\
Illicit drug use & & \\
Previous illicit drug user & 11 & $2.84 \%$ \\
No illicit drug use & 35 & $9.02 \%$ \\
Current illicit/non-prescription drug use & 342 & $88.14 \%$ \\
Currently on treatment for other medical condition & 107 & $27.58 \%$ \\
Substance use to manage work related stress (WRS) & & \\
Feel need to smoke to manage WRS & 103 & $26.55 \%$ \\
Prescription drug use to manage WRS & 65 & $16.75 \%$ \\
Feel need to drink alcohol to manage WRS & 44 & $11.34 \%$ \\
Feel need to use illicit drugs to manage WRS & 16 & $4.13 \%$ \\
Ever diagnosed with a mental health condition & 43 & $11.08 \%$ \\
Currently on treatment for mental health condition & 28 & $7.22 \%$ \\
Family history of mental health condition & 44 & $11.34 \%$ \\
\hline
\end{tabular}

Data are presented as \% or median (interquartile range) unless otherwise indicated. ${ }^{\dagger}$ Cage Score; Define positive score $\geq 2 / 4$ positive responses to CAGE questions on problem drinking. WRS: work-related stress.

\subsection{Occupational History}

Most of the participants worked in operational services (71\%) compared to support services (Table 1). Half worked in a rural setting (53\%). The median years of employment in current role was 7.7 (IQR: 3.4-12.2) while the overall median years employed in a health environment was 9.9 (IQR: 5.8-15). A significant but moderate positive correlation (Spearman rho $0.52, p<0.001$ ) existed between participant age and employment duration in their current role. A similarly positive yet strong correlation (Spearman rho 0.69, $p<0.001$ ) existed between participant age and employment duration in the health sector. A quarter $(25 \%)$ of the participants reported changing jobs in the past five years primarily for better job prospects, fewer for medical reasons.

\subsection{Mental Health Status and Work-Related Stress Management}

The self-reported prevalence of ever being diagnosed with a mental health condition was $11 \%$, with $7 \%$ reporting currently being on treatment for a mental health condition (Table 2). Based on the IES-R questionnaire, the prevalence of PTSD in all of the participants was $30.41 \%$; females had a higher prevalence $(35 \%)$ compared to males $(27 \%)$, but this difference was not statistically significant $(p$-value $=0.085)$. PTSD prevalence in operational staff $(30 \%)$ was also not significantly different $(p$-value $=0.853)$ to that of support services staff $(31 \%)$ (Table 3$)$. 
Table 3. Frequency and distribution of mental health specific variables $(n=388)$.

\begin{tabular}{|c|c|c|}
\hline Participant Characteristics & Frequency $(n)$ & Percentage $(\%)$ \\
\hline \multicolumn{3}{|l|}{ Prevalence of PTSD } \\
\hline All participants & 118 & $30.41 \%$ \\
\hline \multicolumn{3}{|l|}{ Prevalence of PTSD by gender } \\
\hline Males $(n=213)$ & 57 & $26.76 \%$ \\
\hline Females $(n=175)$ & 61 & $34.86 \%$ \\
\hline \multicolumn{3}{|l|}{ Prevalence of PTSD by job category/role } \\
\hline Operational staff $(n=277)$ & 85 & $30.69 \%$ \\
\hline Support staff $(n=111)$ & 33 & $29.73 \%$ \\
\hline \multicolumn{3}{|l|}{ Role limitations due to emotional problems (SF36) } \\
\hline Any emotional problems with regular work in past 4 weeks & 156 & $40.21 \%$ \\
\hline Cut down on amount of time spent on work/other activities & 94 & $24.23 \%$ \\
\hline Accomplished less than would like & 110 & $28.35 \%$ \\
\hline Not working as carefully as usual & 90 & $23.20 \%$ \\
\hline Awareness of services to manage WRS & 277 & $71.39 \%$ \\
\hline \multicolumn{3}{|l|}{ Extent of training on how to manage WRS } \\
\hline No training & 268 & $69.07 \%$ \\
\hline Some training & 110 & $28.35 \%$ \\
\hline Extensive training & 10 & $2.58 \%$ \\
\hline \multicolumn{3}{|l|}{ Extent of training on services available for WRS } \\
\hline No training & 266 & $68.56 \%$ \\
\hline Some training & 111 & $28.61 \%$ \\
\hline Extensive training & 11 & $2.84 \%$ \\
\hline \multicolumn{3}{|l|}{ Barriers to seeking help for WRS } \\
\hline Fear that services are not confidential & 149 & $38.40 \%$ \\
\hline Fear that my career will be negatively affected & 88 & $22.68 \%$ \\
\hline Difficult to get time off from work & 69 & $17.78 \%$ \\
\hline Lack finances or medical aid & 68 & $17.52 \%$ \\
\hline Do not know where to get help & 61 & $15.72 \%$ \\
\hline Difficult to schedule appointment & 48 & $12.37 \%$ \\
\hline Lack transport to access help & 42 & $10.82 \%$ \\
\hline \multicolumn{3}{|l|}{ How services/support for WRS can be improved } \\
\hline Train staff to recognize when stressed & 262 & $67.52 \%$ \\
\hline Have more supportive management & 242 & $63.37 \%$ \\
\hline Address staff shortages & 243 & $62.63 \%$ \\
\hline Provide counselling on premises & 224 & $57.73 \%$ \\
\hline Allow for debriefing or discussion & 219 & $56.44 \%$ \\
\hline Train supervisors to detect WRS & 203 & $52.32 \%$ \\
\hline Improve culture within service & 154 & $39.70 \%$ \\
\hline Provide accessible treatment services & 153 & $39.43 \%$ \\
\hline Provide group coaching & 148 & $38.14 \%$ \\
\hline Lessen workload & 119 & $30.67 \%$ \\
\hline Rotate shifts: work in high and low trauma settings & 86 & $22.16 \%$ \\
\hline Rotate shifts to allow enough rest & 82 & $21.13 \%$ \\
\hline Provide counselling telephonically & 78 & $20.10 \%$ \\
\hline
\end{tabular}

Data are presented as \% or median (interquartile range) unless otherwise indicated. PTSD: post-traumatic stress disorder; WRS: work-related stress.

Forty percent of participants reported having had emotional problems with regular work in the preceding 4 weeks. Substances used by participants to manage WRS include cigarette/tobacco smoking $(27 \%)$, drinking alcohol (11\%), illicit drug use $(4.13 \%)$ and the use of prescription medication (17\%). The majority had not received any specific training on how to manage WRS (69\%) or on what support services were available $(69 \%)$. The greatest barrier encountered in seeking help for WRS is the fear that the services provided by the employer were not confidential (38\%) and that the participant's career would be negatively affected $(23 \%)$. Preferred sources of support for participants include a family member or friend $(63 \%)$, a spouse or partner $(56 \%)$ and a spiritual leader $(41 \%)$ while the least preferred $(20 \%)$ is a trade union or labour representative (Table 4$)$. The participants recommended 
various ways in which services for WRS could be improved (Table 3), including training for staff to recognize when they are stressed (68\%) and more supportive management (63\%), with the least preferred method being telephonic counselling (20\%).

Table 4. Descriptive results for preferred sources of support $(n=388)$.

\begin{tabular}{cc}
\hline Sources of Support & Likely to Seek Support \\
\hline Spouse or partner $(n=336)$ & $62.50 \%$ \\
Family member or friend $(n=345)$ & $55.94 \%$ \\
Spiritual or religious leader $(n=337)$ & $40.95 \%$ \\
Colleague or co-worker $(n=338)$ & $29.29 \%$ \\
Telephonic counsellors $(n=340)$ & $27.94 \%$ \\
Supervisor $(n=339)$ & $25.96 \%$ \\
Employee assistance programme $(n=336)$ & $25.60 \%$ \\
Occupational health/wellness staff $(n=336)$ & $25.30 \%$ \\
Trade union or labour representative $(n=327)$ & $20.18 \%$ \\
\hline
\end{tabular}

\subsection{Occupational and Environmental Risk Factors for PTSD}

Bivariate analysis was undertaken to examine the correlation between post-traumatic stress symptom scores with various workplace risk factors. These associations were all found to be statistically significant (Table 5). Increasing PTSD scores had a significant but moderate positive correlation (Spearman rho 0.56, $p$-value $<0.001$ ) with chronic workplace stress and a weak positive correlation (Spearman rho 0.34, $p$-value $<0.001$ ) with critical incident stress. A significant but weak negative correlation (Spearman rho- $0.25, \mathrm{p}<0.001$ ) was found between PTSD and resilience, and a similar weak negative correlation (Spearman rho-0.22, p-value < 0.006) was found between PTSD and quality of life.

Table 5. Occupational and environmental risk factors for PTSD (Univariate and Bivariate analysis).

\begin{tabular}{ccc}
\hline Risk Factors & Mean (SD) & Correlation with PTSD Score \\
\hline SF36 QoL score (role limitation) * & $37.2(38.42)$ & $-0.22(p=0.006)$ \\
Resilience (CD-RISC score) & $28.0(6.93)$ & $-0.25(p<0.001)$ \\
Operational stress & $33.8(15.27)$ & $0.56(p<0.001)$ \\
Organizational stress & $42.5(16.06)$ & $0.46(p<0.001)$ \\
Chronic workplace stress $^{+}$ & $76.4(28.41)$ & $0.56(p<0.001)$ \\
Critical incident stress $^{\text {Posttraumatic stress }}$ & $27.7(17.01)$ & $0.34(p<0.001)$ \\
& $23.6(22.41)$ & 1
\end{tabular}

* SF36 Quality of Life score (role limitations due to emotional problems). ${ }^{+}$Chronic workplace stress (operational stress and organizational stress combined). CD-RISC: Connor-Davidson Resilience Scale.

Unadjusted logistic regression analysis was performed (Table 6). PTSD was associated with working in operational services (Health-Net) $(\mathrm{OR}=6.00,95 \% \mathrm{CI}$ : 1.32-27.10), needing to smoke to manage WRS (OR $=2.13,95 \% \mathrm{CI}: 1.33-3.41)$, needing to use alcohol to manage WRS (OR = 3.55, 1.87-6.75), alcohol misuse (OR = 2.85, 95\% CI: 1.50-5.44), current illicit drug use $(\mathrm{OR}=4.18,1.20-14.58)$, feeling the need to use illicit drugs $(\mathrm{OR}=4.07,95 \% \mathrm{CI}$ : 1.44-11.48) and the use of prescription medication to manage WRS (OR $=4.63,95 \% \mathrm{CI}$ : 2.65-8.09). Working in a rural area was found to be protective against PTSD (OR = 0.61, 95\% CI: 0.40-0.95).

Participants with a self-reported mental health condition were more likely to have PTSD (OR $=3.76,95 \% \mathrm{CI}: 1.96-7.21)$, including those currently on treatment for a medical condition $(\mathrm{OR}=1.95,95 \% \mathrm{CI}$ : $1.22-3.11)$. Those who had emotional problems with regular work in the past 4 weeks were also more likely to have PTSD (OR $=5.37,95 \%$ CI: 3.36-8.59). PTSD was positively associated with chronic workplace stress, with the strength of association greater in those with moderate $(\mathrm{OR}=4.71,95 \% \mathrm{CI}$ : 2.16-10.30) to high (OR $=20.03$, 95\% CL: 9.33-43.03) levels of chronic workplace stress compared to those with low WRS. Furthermore, PTSD was associated with critical incident stress; those with 
the highest critical incidence stress displayed greater odds of being diagnosed with PTSD $(\mathrm{OR}=3.19,95 \% \mathrm{CI}: 1.88-5.40)$. PTSD was negatively associated with increased quality of life (OR $=0.38,95 \%$ CI: $0.15-0.94)$ and increased resilience score $(\mathrm{OR}=0.26,95 \% \mathrm{CI}$ : $0.14-0.48))$.

Table 6. Unadjusted and adjusted regression analysis of the correlates of PTSD $(\mathrm{N}=388)$.

\begin{tabular}{|c|c|c|c|c|}
\hline \multirow[b]{2}{*}{ Correlates } & \multicolumn{2}{|c|}{ Unadjusted Univariate Analysis \$ } & \multicolumn{2}{|c|}{ Adjusted Multivariate Analysis \$ * } \\
\hline & OR $(95 \%$ CI $)$ & $p$-Value & OR $(95 \% \mathrm{CI})$ & $p$-Value \\
\hline Age $(n=339)$ & & & - & - \\
\hline$\leq 30$ years & 1.00 & 1.00 & & \\
\hline $31-40$ years & $0.98(0.53-1.82)$ & 0.95 & & \\
\hline $41-50$ years & $1.19(0.62-2.30)$ & 0.60 & & \\
\hline$\geq 51$ years & $0.74(0.30-1.84)$ & 0.52 & & \\
\hline \multicolumn{5}{|l|}{ Gender } \\
\hline Male & 1.00 & 1.00 & - & - \\
\hline Female & $1.46(0.95-2.26)$ & 0.09 & - & - \\
\hline \multicolumn{5}{|l|}{ Education } \\
\hline Basic (Grade 1 to 12 ) & 1.00 & 1.00 & - & - \\
\hline Certificate & $1.62(0.71-3.69)$ & 0.26 & - & - \\
\hline Diploma & $0.84(0.29-2.47)$ & 0.76 & - & - \\
\hline Degree & $2.03(0.65-6.34)$ & 0.23 & - & - \\
\hline \multicolumn{5}{|l|}{ Smoking } \\
\hline Non-smoker & 1.00 & 1.00 & 1.00 & 1.00 \\
\hline Ex-smoker & $1.00(0.46-2.20)$ & 0.99 & $1.09(0.46-2.59)$ & 0.841 \\
\hline Current smoker & $1.33(0.83-2.14)$ & 0.23 & $1.76(1.05-2.95)$ & 0.033 \\
\hline \multicolumn{5}{|l|}{ Feel need to smoke to manage WRS } \\
\hline Never feel need & 1.00 & 1.00 & 1.00 & 1.00 \\
\hline Feel need to smoke & $2.13(1.33-3.41)$ & 0.002 & $2.35(1.40-3.93)$ & 0.001 \\
\hline \multicolumn{5}{|l|}{ Alcohol misuse $(n=200)$} \\
\hline No alcohol misuse (CAGE score $0-1$ ) & 1.00 & 1.00 & 1.00 & 1.00 \\
\hline Alcohol misuse (CAGE score 2-4) & $2.85(1.50-5.44)$ & 0.001 & $3.86(1.80-8.23)$ & 0.001 \\
\hline \multicolumn{5}{|l|}{ Feel need to use alcohol to manage WRS } \\
\hline Never feel need & 1.00 & 1.00 & 1.00 & 1.00 \\
\hline Feel need to use alcohol & $3.55(1.87-6.75)$ & $<0.001$ & $6.37(2.93-13.85)$ & $<0.001$ \\
\hline \multicolumn{5}{|l|}{ Drug/illicit substance use } \\
\hline Non-drug user & 1.00 & 1.00 & 1.00 & 1.00 \\
\hline Ex-drug user & $0.95(0.44-2.06)$ & 0.905 & $1.08(0.48-2.41)$ & 0.850 \\
\hline Current drug user & $4.18(1.20-14.58)$ & 0.025 & $16.4(1.87-143.86)$ & 0.012 \\
\hline \multicolumn{5}{|l|}{ Feel need to use illicit drugs to manage WRS } \\
\hline Never feel need & 1.00 & 1.00 & 1.00 & 1.00 \\
\hline Feel need to use drugs & $4.07(1.44-11.48)$ & 0.008 & $5.99(1.74-20.62)$ & 0.005 \\
\hline \multicolumn{5}{|c|}{ Feel need to use prescription drugs to manage WRS } \\
\hline Never feel need & 1.00 & 1.00 & 1.00 & 1.00 \\
\hline $\begin{array}{l}\text { Feel need to use prescription drugs } \\
\text { Job category (by department) }\end{array}$ & $4.63(2.65-8.09)$ & $<0.001$ & $4.51(2.48-8.20)$ & $<0.001$ \\
\hline Managers, Admin, Finance \& Other & 1.00 & 1.00 & 1.00 & 1.00 \\
\hline Ambulance Services & $3.10(0.89-10.7)$ & 0.075 & $2.97(0.82-10.68)$ & 0.096 \\
\hline HealthNet & $6(1.32-27.10)$ & 0.020 & $5.02(0.93-27.11)$ & 0.061 \\
\hline Rescue Services & $0.58(0.09-3.8)$ & 0.571 & $0.78(0.11-5.40)$ & 0.798 \\
\hline Call Centre & $3.44(0.95-12.54)$ & 0.060 & $4.04(1.07-15.21)$ & 0.039 \\
\hline \multicolumn{5}{|l|}{ Work location (within province) } \\
\hline Cape Town metropole & 1.00 & 1.00 & 1.00 & 1.00 \\
\hline Rural areas & $0.61(0.40-0.95)$ & 0.027 & $0.90(0.84-0.97)$ & 0.006 \\
\hline \multicolumn{5}{|l|}{ Mental health diagnosis } \\
\hline Never diagnosed & 1.00 & 1.00 & 1.00 & 1.00 \\
\hline Diagnosed & $3.76(1.96-7.21)$ & $<0.001$ & $3.52(1.78-6.97)$ & $<0.001$ \\
\hline \multicolumn{5}{|l|}{ Treatment for other medical condition } \\
\hline Not currently on treatment & 1.00 & 1.00 & 1.00 & 1.00 \\
\hline Currently on treatment & $1.95(1.22-3.11)$ & 0.005 & $2.19(1.29-3.73)$ & 0.004 \\
\hline
\end{tabular}


Table 6. Cont.

\begin{tabular}{|c|c|c|c|c|}
\hline \multirow[b]{2}{*}{ Correlates } & \multicolumn{2}{|c|}{ Unadjusted Univariate Analysis \$ } & \multicolumn{2}{|c|}{ Adjusted Multivariate Analysis ${ }^{\text {\$ }}$} \\
\hline & OR $(95 \% \mathrm{CI})$ & $p$-Value & OR $(95 \% \mathrm{CI})$ & $p$-Value \\
\hline \multicolumn{5}{|l|}{$\begin{array}{l}\text { Emotional problems with regular work } \\
\text { (past } 4 \text { weeks) }\end{array}$} \\
\hline No emotional problems with regular work & 1.00 & 1.00 & 1.00 & 1.00 \\
\hline \multicolumn{5}{|l|}{ SF36 QoL score (role limitation) } \\
\hline SF36 QoL score $(\leq 3)$ & 1.00 & 1.00 & 1.00 & 1.00 \\
\hline SF36 QoL score (4) & $0.44(0.18-1.07)$ & 0.071 & $0.33(0.12-0.87)$ & 0.024 \\
\hline SF36 QoL score (5) & $0.33(0.14-0.78)$ & 0.012 & $0.26(0.10-0.69)$ & 0.007 \\
\hline SF36 QoL score (6) & $0.38(0.15-0.94)$ & 0.036 & $0.34(0.12-0.96)$ & 0.042 \\
\hline \multicolumn{5}{|l|}{ Resilience (CD-RISC score) } \\
\hline CD-RISC score $(\leq 26)$ & 1.00 & 1.00 & 1.00 & 1.00 \\
\hline CD-RISC score (27-32) & $0.56(0.34-0.91)$ & 0.019 & $0.67(0.40-1.15)$ & 0.148 \\
\hline CD-RISC score (33-40) & $0.26(0.14-0.48)$ & $<0.001$ & $0.29(0.15-0.57)$ & $<0.001$ \\
\hline \multicolumn{5}{|l|}{ Chronic workplace stress ${ }^{\dagger}$ (CSQ score) } \\
\hline CSQ score $(\leq 63)$ & 1.00 & 1.00 & 1.00 & 1.00 \\
\hline CSQ score (64-88) & $4.71(2.16-10.30)$ & $<0.001$ & $4.46(1.93-10.31)$ & $<0.001$ \\
\hline CSQ score (89-140) & $20.03(9.33-43.03)$ & $<0.001$ & 19.83(8.75-44.89) & $<0.001$ \\
\hline \multicolumn{5}{|l|}{ Critical incident stress (CII score) } \\
\hline CII score $(\leq 18)$ & 1.00 & 1.00 & 1.00 & 1.00 \\
\hline CII score (19-37) & $1.14(0.64-2.03)$ & 0.666 & $1.28(0.66-2.48)$ & 0.468 \\
\hline CII score (38-72) & $3.19(1.88-5.40)$ & $<0.001$ & $4.27(2.24-8.15)$ & $<0.001$ \\
\hline
\end{tabular}

* Data adjusted for age, gender and education. $\$$ Statistically significant results indicated in bold ${ }^{\dagger}$ Chronic workplace stress (operational stress and organizational stress combined). CD-RISC: Connor-Davidson Resilience Scale. WRS: work-related stress.

Multivariate regression analysis was computed (Table 6), with adjustment for age, gender and educational level. PTSD was associated with being a current smoker $(\mathrm{OR}=1.76$, 95\% CI: 1.05-2.95) (previously non-significant) and feeling the need to smoke to manage WRS. Some associations between PTSD and various risk factors were strengthened following adjusted analysis. These included: the need to use alcohol to manage WRS (OR = 6.37, CI: 2.93-13.85), alcohol misuse (OR = 3.86, 95\% CI: 1.80-8.23), current illicit drug use (OR $=16.4,95 \%$ CI: 1.87-143.86) and feeling the need to use illicit drugs (OR $=5.99$, 95\% CI: 1.74-20.62). The association between PTSD and working in operational services (Health-Net) was no longer significant. However, PTSD was now associated with working in the call centre (OR $=4.04,95 \% \mathrm{CI}: 1.07-15.21)$. Increased exposure to chronic and critical workplace stress continued to be positively associated with PTSD while increasing levels of quality of life and resilience were protective.

\section{Discussion}

The prevalence of PTSD in this study was found to be 30\%, which is higher than that reported for the South African general population (2.3\% lifetime prevalence) [28] and reported globally $(1.0-4.3 \%)$ [29]. It is also higher than estimated by previous studies conducted in the Western Cape, which found a prevalence of $6.7 \%$ in ambulance personnel $(n=99)$ and $16 \%(n=131)$ in paramedic trainees $[11,12]$. When compared to other lowermiddle-income countries (LMICs), PTSD prevalence in this study is higher than the $13.6 \%$ reported for Egyptian paramedics $(n=140)$ and $15 \%$ in Brazilian ambulance workers $(n=234)[30,31]$. Systematic reviews and meta-analyses from studies conducted in highincome countries (HICs) report a prevalence as low as $11 \%$ [9] and as high as $20 \%$ among ambulance personnel [32].

The higher prevalence found in this study can be explained by differences in study population, context and methodology. In the Western Cape Province, as of 2014, an escalation in criminal attacks was experienced by ambulance personnel (including threats, 
verbal/physical assaults, robbery/theft, random stoning), with likely increases in chronic stress and critical incident stress exposure [33]. To mitigate the potential impact of these traumatic exposures on ambulance personnel, certain areas within the Cape Town Metropole were designated as 'Red Zones', requiring a police escort be made available before ambulance personnel go into an area to assist patients [33]. Previous studies undertaken before 2014 used a different PTSD scoring scale and had relatively smaller sample sizes than this study. Given the escalation in attacks on paramedics since 2014, the participants in this study may be at greater risk for PTSD and more likely to have concerns regarding PTSD and a need to report their symptoms compared to non-participants [13].

Of note in the current study is that the PTSD prevalence in operational staff $(30 \%)$ consisting of ambulance personnel on the road was not significantly different ( $p$-value $=0.853$ ) to that of support services staff $(31 \%)$ consisting largely of call center workers $(n=88)$ and managers/staff in various administrative roles $(n=33)$. The similar PTSD prevalence may be because of the relocation or accommodation of operational staff previously exposed to trauma to a call centre or management role due to mental or physical health or reasons of incapacity. The lack of skills in handling the secondary trauma related to working (telephonically) with families or patients in distress may also be independently associated with PTSD status. It has been noted that, although removed from the scene of the incident, emergency service call-takers and dispatchers experience similar mental health challenges as front-line ambulance personnel. [34].

Current mental health problems have been demonstrated as a predictor of PTSD [35,36]. Kerai et al. [16] found that emergency personnel with anxiety and depression had higher levels of post-traumatic stress symptoms. Those with a pre-existing mental health condition may be predisposed to developing PTSD in the presence of chronic and acute stress events associated with first responder work [2,21,37]. Participants who reported having a mental health condition were more likely to have PTSD and more likely to report emotional problems in the past 4 weeks with role limitations due to emotional problems. These findings were consistent with those reported in Brazilian paramedics [31], with the mean (SD) QOL score for role limitation due to emotional problems in our study being 37.2 (IQR: 38.42), which was lower than that for Brazilian paramedics with PTSD, which was 47.2 (IQR: 33.21). This indicates an even poorer quality of life in our population. Poorer quality of life score was also associated with increased odds of PTSD diagnosis. Participants with PTSD were also more likely to report being on treatment for a medical condition; this is a phenomenon also observed in Brazilian paramedics with PTSD as they had more medical visits and hospital admissions in the previous 12 months compared to those without PTSD.

Increased levels of exposure to chronic and critical incident stress were significantly associated with PTSD diagnosis, with those exposed to the highest levels having greater odds of being diagnosed compared to those with lower exposures, reflecting a doseresponse relationship. The mean critical incident score (27.7) found in this study was higher than that previously found (20.59, 95\% CI: 19.11 to 22.06$)$ in this population $[6,24]$, which is indicative of not only high exposure but a possible increase in critical incident stress over time. Chronic organizational and operational stress both individually and in combination are significant correlates of PTSD in this population. The mean (SD) operational stress score of 33.8 (15.27) was comparable to that found by Donnelley et al. [7] of 31.4 (12.1); while the mean (SD) organizational stress score of 42.5 (16.06) vs. 34.8 (13.5) was higher (13.5). These findings demonstrate high chronic stress exposure in the population surveyed and importantly highlight the potential impact organizational factors such as staff shortages have on PTSD prevalence. Staff shortages, for example, could result in ambulance personnel experiencing more frequent exposure to critical incidents and resultant increases in chronic stress levels. In this study, however, individuals who worked in a rural area were less likely to develop PTSD. This may reflect a workload with less exposure to chronic stress or less critical incident stress events compared to those working in urban settings. 
The use of substances to manage or cope with WRS deserves further exploration. In this study, the need to smoke, drink alcohol and to use illicit drugs to manage WRS was associated with PTSD. Participants displayed high rates of problem drinking (27\% overall, $32.03 \%$ in males and $18.06 \%$ in females). This was higher than that for paramedic trainees in the Western Cape (23\%) and the general population $(16 \%$ in men and $3 \%$ in women) [12,38]. The use of prescription drugs such as anxiolytics and antidepressants by $17 \%$ of the participants to manage WRS was also significantly associated with PTSD. This was higher than the $5.6 \%$ personal medication use reported in emergency medical service personnel $(n=518)$ in Pakistan [16]. While it is not clear whether the substance use is because of PTSD or preceded it, this places participants at risk of dual psychiatric diagnoses (substance use disorder as well as PTSD), which is more difficult to manage and has greater morbidity associated with it $[17,21]$.

Participants indicated that they are most likely to seek help/support for WRS from a spouse or partner $(62.50 \%)$ and family member or friend $(55.94 \%)$, which highlights the important role that family and friends can play when formulating interventions aimed at reducing WRS. Canadian paramedics were similarly more likely to seek support from a family member or friend (81.4\%) and a regular work partner (73.2\%) [7]. Surprisingly, however, there was a lower likelihood of seeking support from employer-provided services, with only $25 \%$ of participants (compared to $38.6 \%$ in Canadian paramedics) likely to use such a service. Various barriers to accessing support included fear that services are not confidential and that participant's career will be negatively affected. Difficulty in getting time off to access care and a lack of finances/medical aid are other barriers faced.

The participants identified the need for more training of staff in recognizing WRS, having a more supportive management, addressing staff shortages and provision of counseling on the work premises as preferred measures to help reduce WRS. These correlate with the chronic organizational stressors already discussed and highlights the extent to which these are concerning to the participants. Of note is that the majority $(69 \%)$ reported having not received any specific training on how to manage WRS or on what services are available (69\%). Minnie et al. [5] also found that 72\% of ambulance personnel noted having received little or no training on managing the emotional effects of exposure to traumatic effects. Promoting resilience in ambulance personnel could also be helpful in mitigating the challenges related to the work of ambulance personnel [39], as reflected in a negative correlation between PTSD and resilience scores. Those with the highest resilience scores were less likely to be diagnosed with PTSD than those with lower scores. Similar studies have shown that lower resilience was significantly associated with PTSD status [12,39]. While resilience training helps equip emergency workers to deal with traumatic events, other interventions, such as access to psychiatric screening by trained mental health professionals for earlier detection of PTSD symptoms and provision of evidence-based treatments, should be considered [39-41]. Broader organizational and operational concerns raised such as a supportive work environment and follow-up of at-risk workers should be addressed to help prevent the development of PTSD from work-related trauma $[40,41]$.

\section{Limitations}

The study had several limitations. The findings are based on 388 respondents from a workforce of 2000 as it relied on voluntary participation. Ambulance personnel who chose not to participate may be different to those who did, which may alter the overall PTSD prevalence obtained, a reflection of selection bias. Reasons for lack of participation include possible stigma relating to a mental health topic and 'response fatigue' as other surveys were being conducted during the time of this study. Mistrust and concerns that the questionnaire would not be confidential or would negatively affect one's work prospects may also be a contributing factor. However, this was mitigated by meeting trade union representatives and staff at ambulance bases to explain the study methodology and data handling concerns. 
Not all of the potential risk factors for PTSD were assessed to help keep the questionnaire to an acceptable length. Questions on childhood trauma, the full SF36 quality of life questionnaire and other mental health screening tools could not be included. Due to resource limitations, the study was unable to use the gold standard test for the diagnosis of PTSD (clinician-based diagnostic interview); however, validated PTSD screening tools were used. Social desirability bias may have occurred when participants responded in a socially acceptable manner (e.g., underreporting mental health symptoms and substance use), which could have led to reduced study estimates. Recall bias may also have arisen as the questionnaire required participants to rate symptoms based on a traumatic event that was experienced up to 6 months earlier. There was no control group from a general population whose findings could be compared to those of the ambulance personnel. Further, studies of occupational groups are vulnerable to the healthy worker effect, which may have led to an underestimate of PTSD as those severely impaired by PTSD may have left their employ, leaving a generally healthier population behind. The cross-sectional study design further limits the inference of causal associations as data on both exposures and outcomes were collected simultaneously. Concordance with the findings from other studies and biological plausibility do, however, suggest that significant associations may be reflecting potential risk factors implicated in the development of PTSD in this population.

\section{Conclusions}

PTSD prevalence was markedly higher in ambulance personnel than in the general population, reflecting an increased risk in this occupational grouping. This is a cause for concern. Those with a mental health condition who were receiving treatment for a medical condition, who self-reported substance use and abuse, who were working in an urban area or who were exposed to moderate to high chronic and critical incident stress were at increased risk. Support from family and friends including co-workers could form an integral part of interventions designed to support ambulance personnel. Greater attention needs to be paid to the barriers that have been identified as limiting access to available support for work-related stress. In this regard, efforts should be aimed at ensuring access to confidential and acceptable mental health services that are provided in a non-punitive manner without leaving workers out of pocket. The high rates of organizational and workrelated stress experienced by this population and the strong associations between PTSD and substance use offer starting points for interventions aimed at reducing the burden and impact of PTSD.

Further studies using clinician-based diagnostic interviews are recommended and research into evidence-based methods of screening and managing ambulance personnel for PTSD and the development of appropriate interventions and protocols for lower-middleincome countries context are needed.

Author Contributions: S.A. was responsible for the overall study design and overseeing the analysis and write up. I.N. was responsible for the study design, conducting the fieldwork, data analysis, preparation and submission of the manuscript. All authors have read and agreed to the published version of the manuscript.

Funding: This research received no external funding.

Institutional Review Board Statement: The study was conducted in accordance with the Declaration of Helsinki and approved by the Human Research Ethics Committee of the University of Cape Town (HREC 517/2019) prior to the study being conducted. All participants in the study provided written informed consent.

Informed Consent Statement: Informed consent was obtained from all participants involved in the study.

Data Availability Statement: The data presented in this study are available on request from the corresponding author. The data are not publicly available due to legal and privacy issues. 


\begin{abstract}
Acknowledgments: We would like to acknowledge the ambulance staff and employees of the Western Cape Department of Health that participated in this study. We would also like to acknowledge the support provided by the management, employee wellness and occupational health department of the Western Cape Department of Health Emergency Medical Services.
\end{abstract}

Conflicts of Interest: The authors declare no conflict of interest.

\title{
References
}

1. Roberts, M.H.; Sim, M.R.; Black, O.; Smith, P. Occupational injury risk among ambulance officers and paramedics compared with other healthcare workers in Victoria, Australia: Analysis of workers' compensation claims from 2003 to 2012. Occup. Environ. Med. 2015, 72, 489-495. [CrossRef] [PubMed]

2. Donnelly, E.; Siebert, D. Occupational Risk Factors in the Emergency Medical Services. Prehospital Disaster Med. 2012, 24, 422-429. [CrossRef] [PubMed]

3. Taylor, J.A.; Davis, A.L.; Barnes, B.; Lacovara, A.V.; Patel, R. Injury risks of EMS responders: Evidence from the National Fire Fighter Near-Miss Reporting System. BMJ Open 2015, 5, 1-8. [CrossRef]

4. European Agency for Safety and Health at Work (EU-OSHA). Emergency Services: A Literature Review on Occupational Safety and Health Risks; Publications of the Office European Union: Luxembourg, 2011. [CrossRef]

5. Minnie, L.; Goodman, S.; Wallis, L. Exposure to daily trauma: The experiences and coping mechanism of Emergency Medical Personnel: A cross-sectional study. Afr. J. Emerg. Med. 2015, 5, 12-18. [CrossRef]

6. Ward, C.L.; Lombard, C.J.; Gwebushe, N. Critical incident exposure in South African emergency services personnel: Prevalence and associated mental health issues. Emerg. Med. J. 2006, 23, 226-231. [CrossRef] [PubMed]

7. Donnelly, E.A.; Bradford, P.; Davis, M.; Hedges, C.; Klingel, M. Predictors of posttraumatic stress and preferred sources of social support among Canadian paramedics. CJEM 2016, 18, 205-212. [CrossRef]

8. Berger, W.; Coutinho, E.S.F.; Figueira, I.; Marques-Portella, C.; Luz, M.P.; Neylan, T.C.; Marmar, C.R.; Mendlowicz, M.V. Rescuers at risk: A systematic review and meta-regression analysis of the worldwide current prevalence and correlates of PTSD in rescue workers. Soc. Psychiatry Psychiatr. Epidemiol. 2012, 47, 1001-1011. [CrossRef]

9. Petrie, K.; Milligan-Saville, J.; Gayed, A.; Deady, M.; Phelps, A.; Dell, L.; Forbes, D.; Bryant, R.A.; Calvo, R.A.; Glozier, N.; et al. Prevalence of PTSD and common mental disorders amongst ambulance personnel: A systematic review and meta-analysis. Soc. Psychiatry Psychiatr. Epidemiol. 2018, 53, 897-909. [CrossRef]

10. Brysiewicz, P. Trauma in South Africa. Int. J. Trauma Nurs. 2001, 7, 129-132. [CrossRef]

11. Stewart, J.; Swartz, L. Posttraumatic Stress Symptoms in Emergency Service Ambulance Personnel. Soc. Work/Maatskaplike Werk 2014, 41, 362-377. [CrossRef]

12. Fjeldheim, C.B.; Nöthling, J.; Pretorius, K.; Basson, M.; Ganasen, K.; Heneke, R.; Cloete, K.J.; Seedat, S. Trauma exposure, posttraumatic stress disorder and the effect of explanatory variables in paramedic trainees. BMC Emerg. Med. 2014, 14, 11. [CrossRef] [PubMed]

13. Rybojad, B.; Aftyka, A.; Baran, M.; Rzonca, P. Risk Factors for Posttraumatic Stress Disorder in Polish Paramedics: A Pilot Study. J. Emerg. Med. 2016, 50, 270-276. [CrossRef] [PubMed]

14. Jonsson, A.; Segesten, K. Daily stress and concept of self in Swedish ambulance personnel. Prehospital Disaster Med. 2004, 19, 226-234. [CrossRef] [PubMed]

15. Bennett, P.; Williams, Y.; Page, N.; Hood, K.; Woollard, M. Levels of mental health problems among UK emergency ambulance workers. Emerg. Med. J. 2004, 21, 235-236. [CrossRef]

16. Kerai, S.M.; Khan, U.R.; Islam, M.; Asad, N.; Razzak, J.; Pasha, O. Post-traumatic stress disorder and its predictors in emergency medical service personnel: A cross-sectional study from Karachi, Pakistan. BMC Emerg. Med. 2017, 17, 26. [CrossRef] [PubMed]

17. Klein, S.; Alexander, D.A. Epidemiology and presentation of post-traumatic disorders. Psychiatry 2009, 8, 282-287. [CrossRef]

18. Pai, A.; Suris, A.M.; North, C.S. Posttraumatic Stress Disorder in the DSM-5: Controversy, Change, and Conceptual Considerations. Behav. Sci. 2017, 7, 7. [CrossRef]

19. American Psychiatric Association. Diagnostic and Statistical Manual of Mental Disorders, 5th ed.; American Psychiatric Association: Arlington County, Virginia, 2013. [CrossRef]

20. van der Ploeg, E.; Kleber, R.J. Acute and chronic job stressors among ambulance personnel: Predictors of health symptoms. Occup. Environ. Med. 2003, 60, 40-46. [CrossRef] [PubMed]

21. Jones, S. Describing the Mental Health Profile of First Responders: A Systematic Review. J. Am. Psychiatr. Nurses Assoc. 2017, 23, 200-214. [CrossRef] [PubMed]

22. Brewin, C.R. Systematic review of screening instruments for adults at risk of PTSD. J. Trauma. Stress 2005, 18, 53-62. [CrossRef]

23. Campbell-Sills, L.; Stein, M.B. Psychometric analysis and refinement of the connor-davidson resilience scale (CD-RISC): Validation of a 10-item measure of resilience. J. Trauma. Stress Off. Publ. Int. Soc. Trauma. Stress Stud. 2007, 20, 1019-1028. [CrossRef] [PubMed]

24. Donnelly, E.A.; Bennett, M. Development of a critical incident stress inventory for the emergency medical services. Traumatol. Int. J. 2014, 20, 1. [CrossRef] 
25. Donnelly, E.A.; Chonody, J.; Campbell, D. Measuring chronic stress in the emergency medical services. J. Workplace Behav. Health 2014, 29, 333-353. [CrossRef]

26. Chen, Y.S.; Chen, M.C.; Chou, F.H.; Sun, F.C.; Chen, P.C.; Tsai, K.Y.; Chao, S.S. The relationship between quality of life and posttraumatic stress disorder or major depression for firefighters in Kaohsiung, Taiwan. Quality Life Res. 2007, 16, 1289-1297. [CrossRef]

27. Lins, L.; Carvalho, F.M. SF-36 total score as a single measure of health quality of life: Scoping review. Sage Open Med. 2016, 4, 1-12. [CrossRef]

28. Stein, D.J.; Seedat, S.; Herman, A.; Moomal, H.; Heeringa, S.G.; Kessler, R.C.; Williams, D.R. Lifetime prevalence of psychiatric disorders in South Africa. Br. J. Psychiatry 2008, 192, 112-117. [CrossRef] [PubMed]

29. Koenen, K.C.; Ratanatharathorn, A.; Ng, L.; McLaughlin, K.A.; Bromet, E.J.; Stein, D.J.; Karam, E.G.; Meron Ruscio, A.; Benjet, C.; Scott, K.; et al. Posttraumatic stress disorder in the World Mental Health Surveys. Psychol. Med. 2017, 47, 2260-2274. [CrossRef] [PubMed]

30. Khashaba, E.O.; El-Sherif, M.A.F.; Ibrahim, A.A.; Neatmatallah, M.A. Work-Related Psychosocial Hazards Among Emergency Medical Responders (EMRs) in Mansoura City. Indian J. Community Med. 2014, 39, 103-110. [CrossRef] [PubMed]

31. Berger, W.; Figueira, I.; Maurat, A.M. Partial and full PTSD in Brazilian ambulance workers: Prevalence and impact on health and on quality of life. J. Trauma. Stress 2007, 20, 637-642. [CrossRef]

32. Sterud, T.; Ekeberg, Ø.; Hem, E. Health status in the ambulance services: A systematic review. BMC Health Serv. Res. 2006, 6, 82. [CrossRef] [PubMed]

33. Brady, L.; De Vries, S.; Gallow, R.; George, A.; Gilson, L.; Louw, M.; Martin, A.W.; Shamis, K.; Stuart, T. Paramedics, poetry, and film: Health policy and systems research at the intersection of theory, art, and practice. Human Resour. Health 2019, 17, 1-6. [CrossRef] [PubMed]

34. Smith, E.C.; Holmes, L.; Burkle, F.M. Exploring the physical and mental health challenges associated with emergency service call-taking and dispatching: A review of the literature. Prehospital Disaster Med. 2019, 34, 619-624. [CrossRef] [PubMed]

35. Brewin, C.R.; Andrews, B.; Valentine, J.D. Meta-analysis of risk factors for posttraumatic stress disorder in trauma-exposed adults. J. Consult. Clin. Psychol. 2000, 68, 748-766. [CrossRef]

36. Ozer, E.J.; Best, S.R.; Lipsey, T.L.; Weiss, D.S. Predictors of posttraumatic stress disorder and symptoms in adults: A meta-analysis. Psychol. Trauma Theory Res. Pract. Policy 2008, 129, 52-73. [CrossRef]

37. Carmassi, C.; Gesi, C.; Corsi, M.; Cremone, I.M.; Bertelloni, C.A.; Massimetti, E.; Olivieri, M.C.; Conversano, C.; Santini, M.; Dell'Osso, L. Exploring PTSD in emergency operators of a major University Hospital in Italy: A preliminary report on the role of gender, age, and education. Ann. Gen. Psychiatry 2018, 17, 1-7. [CrossRef]

38. National Department of Health (NDoH); Statistics South Africa (Stats SA); South African Medical Research Council (SAMRC); ICF. South Africa Demographic and Health Survey, 2016; SADHS: Pretoria, South Africa, 2019.

39. Gayton, S.D.; Lovell, G.P. Resilience in ambulance service paramedics and its relationships with well-being and general health. Traumatology 2012, 18, 58-64. [CrossRef]

40. Lee, W.; Lee, Y.R.; Yoon, J.H.; Lee, H.J.; Kang, M.Y. Occupational post-traumatic stress disorder: An updated systematic review. BMC Public Health 2020, 20, 1-2. [CrossRef]

41. Qian, J.; Wang, W.; Sun, S.; Liu, L.; Sun, Y.; Yu, X. Interventions to reduce post-traumatic stress disorder symptoms in health care professionals from 2011 to 2021: A scoping review. BMJ Open 2022, 12,1-8. [CrossRef] 